\title{
Wish list for 2009
}

Nature Medicine asked leading experts to describe a research breakthrough they hope to see in the next year:

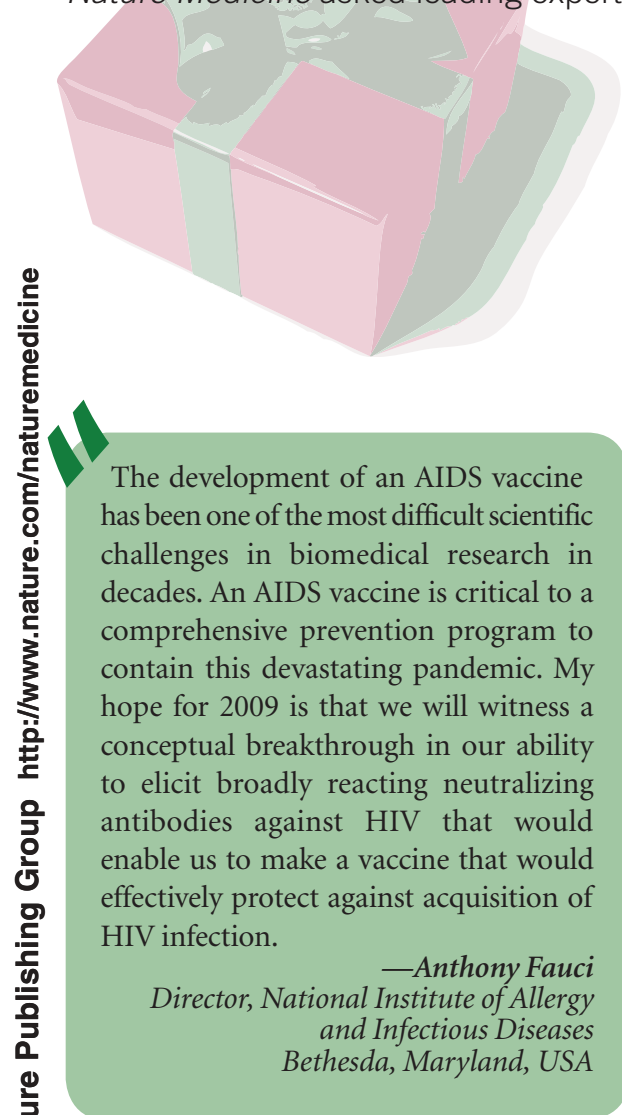

I'd love to see a breakthrough in our understanding of bacterial cell death. Once we know more about the mechanisms and processes involved, we'll be in a far better position to develop therapies for tuberculosis that can dramatically shorten the duration of treatment, which currently takes six months.

$$
\text { -Valerie Mizrahi }
$$

Director, Molecular Mycobacteriology Research Unit, University of the Witwatersrand

Johannesburg, South Africa
N generation of genet in which advances in stem cell research, cellular reprogramming, genomics and computational biology are combined to reveal the causal effects of naturally occurring human genetic variation on a wide range of molecular and cellular phenotypes. Efficient and costeffective strategies should be developed to expand our knowledge of how the human genome functions in health and disease and to stimulate targeted therapies of molecular pathways that will undoubtedly cut across current disease classifications.

$$
\begin{array}{r}
- \text { Elizabeth Nabel } \\
\text { Director, National Heart, Lung, and } \\
\text { Blood Institute } \\
\text { Bethesda, Maryland, USA }
\end{array}
$$

In 2009, I would like to see progress toward solving a major problem that lies at the core of the mission of Harvard Medical School and other such institutions. We do not yet have an optimal approach to leveraging the enormous intellectual assets of researchers in the lab and clinic so as to most effectively bring transformative therapies that are safe and effective to the public. To achieve this goal may require creation of new academic models for therapeutic discovery, new models for relationships with industry that protect and enhance the values and needs of all parties and new approaches to regulation and public policy.

$$
\begin{array}{r}
\text {-Jeffrey Flier } \\
\text { Dean of the Faculty of Medicine, Harvard } \\
\text { Medical School } \\
\text { Boston, Massachusetts, USA }
\end{array}
$$

In 2009, I would most like to see some of the novel approaches that the entire field has begun to explore in Alzheimer's disease and schizophrenia provide results that open up a path to more effective treatments for these disabling disorders that, until now, have been so intractable. Every year, as our research progresses, I am more hopeful that we are getting closer to this goal.

-David Michelson

Vice President, Clinical Neuroscience and Ophthalmology, Merck \& Co. North Wales, Pennsylvania, USA

Today, there is doubt that new molecular therapeutics are substantially changing cancer care and doubt that the fundamental genetic alterations and the unveiling of the molecular principles driving carcinogenesis will intersect with therapy. We have forgotten that efficacious therapy requires maximal target inhibition, that side effects can be ameliorated by scheduling and that personalized medicine includes personalized dosing. We have built a cumbersome system for finding incremental changes in patient benefit that does not enable the discovery of lifechanging combinations. Nonetheless, the treatment of cancer is changing before us. When asked "can we cure cancer?" let us not retreat from the answer "yes, we can."

-William Sellers
Global Head of Oncology, Novartis
Institutes for Biomedical Research
Cambridge, Massachusetts, USA

\section{NOTABLE ADVANCES IN THE CLINIC}

Statins might protect against heart attacks, stroke and death even in people who have low cholesterol, according to results from a study involving nearly 18,000 subjects who had high levels of a C-reactive protein, which indicates inflammation. During the two-year trial, those subjects taking statins were almost $50 \%$ less likely to suffer a stroke or require either angioplasty or bypass surgery (N. Engl. J. Med. doi:10.1056/NEJMoa0807646; 2008). Experts cautioned, however, that the long-term effects of statins are not fully clear and that tests for C-reactive protein levels are not cheap.

A study conducted in Russia found that a drug formerly approved as an antihistamine seemed to improve cognitive function in individuals with Alzheimer's disease. In the study involving 183 subjects, those receiving the placebo had a higher rate of worsening Alzheimer's symptoms than their counterparts who received the medication Dimebon (Lancet 372, 207-215; 2008).

A drug used for years to treat high blood pressure, losartan, could potentially lessen the dangers associated with Marfan syndrome, a genetic disorder of the connective tissue that can cause life-threatening heart problems. In a preliminary trial involving 18 children with Marfan syndrome, the drug decreased the average rate of annual aortic enlargement-a risky heart change-from 3.5 millimeters in diameter to less than 0.5 millimeters (N. Engl. J. Med. 358, 2787-2795; 2008). 